\title{
Editorial of the evolving intelligent applications in engineering special issue
}

\author{
Lazaros Iliadis $^{1}$ - Ilias Maglogiannis ${ }^{2}$
}

Published online: 11 August 2020

○) Springer-Verlag GmbH Germany, part of Springer Nature 2020

The basic characteristic of the twenty-first century postmodern societies is evolution and adaptivity. As a matter of fact, scientific research on Adaptive algorithms and systems using hybrid approaches, is growing overtime. This is the Evolving Intelligent Applications in Engineering Special Issue, of the Springer EVOS Journal. Totally thirteen (13) high quality papers discussing timely, interesting and novel approaches, have been accepted for publication after passing through a peer review process.

The first paper "Freight Transportation Broker Agent Based on Constraint Logic Programming", is authored by Costin Badica from the University of Craiova Romania, Florin Leon, from the Technical University "Gheorghe Asachi" of Iasi Romania and Amelia Badica, from the University of Craiova, Romania. This research paper introduces an agent-based freight brokering system which provides an intelligent logistics brokerage service. It focuses on the transport activity for the efficient allocation of transport resources to the transport applications. Its main contribution is the development of a knowledge-based freight broker, based on agents and constraint programming. The core of the system is a novel declarative optimization model as a part of an optimization agent. The implementation and evaluation of the system were performed using the ECLiPSe-CLP engine.

The second paper is authored by Doina Logofatou, Christina Anderson and Kristiyan Balabanov, from Frankfurt University of Applied Sciences, Frankfurt am Main,

Lazaros Iliadis

liliadis@civil.duth.gr

Ilias Maglogiannis

imaglo@unipi.gr

1 Lab of Mathematics and Informatics (ISCE), Department of Civil Engineering, School of Engineering, Democritus University of Thrace, University Campus, Kimmeria, 67100 Xanthi, Greece

2 Department of Digital Systems, University of Piraeus, Karaoli and Dimitriou 80, 18534 Piraeus, Greece
Germany, Gil Sobol, from Israel Institute of Technology, Haifa, Israel, Daniel Stamate, from Goldsmiths College, University of London, UK and Tymoteusz Cejrowski, from Gdansk University of Technology, Gdansk, Poland. The title is "Particle Swarm Optimization Algorithms for Autonomous Robots with Deterministic Leaders Using Space Filling Movements". The actual goal achieved by this effort is the combination of G. Reynolds' swarm behavior principles with deterministic traits, by using leaders with motions. This effort is based on space filling curves like the ones of Peano and Hilbert (P\&H). Various P\&H combinations with swarm approaches are tested in good practical applications.

Georgios Drakopoulos, from the Ionian University, Corfu, Greece, Panagiotis Gourgaris and Andreas Kanavos, from University of Patras, Greece are the authors of the third paper. It is entitled "Graph Communities in Neo4j Four Algorithms at Work". This paper outlines the implementation of four major community discovery algorithms, namely the Newman Girvan or Edge Betweeness, the Walktrap, the Louvain, and the Clauset, Newman, and Moore (known as CNM) as Java analytics over Neo4j (a popular graph database). Their correctness, in terms of successful community discovery, was evaluated through purely functional metrics applied to two real Twitter graphs.

An interesting research entitled "Weakly Supervised Multilabel Classification for Semantic Interpretation of Endoscopy Video Frames" is authored by Michael D Vasilakakis, Dimitris Diamantis, Evaggelos Spyrou, Dimitris Iakovidis, from the University of Thessaly, Lamia, Greece and Anastasios Kalaouzidis, from the Royal Infirmary of Edinburgh, UK. This research paper addresses the problem of abnormality detection in medical images using computer-based systems. In the context of gastrointestinal video-endoscopy, addressed in this study, the semantics of the normal contents of the endoscopic video frames include normal mucosal tissues, bubbles, debris and the hole of the lumen, whereas the abnormal video frames may include additional semantics corresponding to lesions or blood. This observation 
motivated the authors to investigate various multi-label classification methods, aiming to a richer semantic interpretation of the endoscopic images.

The fifth paper is on "A versatile package recommendation framework aiming at preference score maximization" by Panagiotis Kouris, Georgios Alexandridis, Andreas Stafylopatis National Technical University of Athens, Greece and Iraklis Varlamis, Harokopio University of Athens, Greece. This research introduces a flexible framework for recommending packages that best fit the preferences of the users and that satisfy constraints on the set of the valid packages. The goal has been achieved by developing models of the relations among items and their corresponding categories. Every user has been recommended top k packages. Six recommendation datasets have been used for testing and the performance has been proved very promising.

Mario Malcangi, Hao Quan, from the Universita degli Studi di Milano, Emanuele Vaini, Prospero Lombardi and Marco Di Rienzo from the Fondazione don Gnocchi-Milano, are the authors of the sixth paper. The title is "Evolving Fuzzy-Neural Paradigm applied to the Recognition and Removal of Artefactual Beats in Continuous Seismocardiogram Recordings". Seismocardiogram (SCG) recording is a novel method for the prolonged monitoring of the cardiac mechanical performance during spontaneous behavior. This research paper introduces an Evolving Fuzzy Neural Network (EFuNN) paradigm for the automatic artifact prediction in an SCG signal. More specifically, fuzzy logic has been employed to model human expertise knowledge, using the learning capabilities of an artificial neural network. The evolving capability of the proposed system, has been applied to solve the issue of the physiological variability of the SGC waveform.

An interesting research entitled "Self-Supervised Autoencoders for Clustering and Classification" is described in the seventh paper. The authors are Paraskevi Nousi and Anastasios Tefas from the Aristotle University of Thessaloniki, Greece. This research paper introduces Autoencoders (AUE) for dimensionality reduction. AUE are combined in a hybrid approach with k-means clustering in order to learn lowdimensional representations and to achieve better clustering performance. In fact, this is done through an enhancement of intra-cluster relationships and suppressing inter-cluster ones, in a self-supervised manner. The approach has been tested by considering several datasets related to handwritten digits, objects and faces, and it has proven to improve external cluster quality measuring criteria.

The eighth paper is entitled "A Method for the Detection of the Most Suitable Fuzzy Implication for Data Applications" and it is authored by Panagiotis Pagouropoulos from the Democritus University of Thrace Greece, Christos Dimitrios Tzimopoulos from the Aristotle University of Thessaloniki, Greece and Basil Papadopoulos from the Democritus
University of Thrace Greece. It is a very interesting paper with both theoretical foundations and good practical application. Fuzzy Implications (FI) are widely used in cases where propositional logic is applicable. However, the existence of several FI calls for the selection of the optimal one for each specific case. This research paper introduces a method for detecting the most suitable fuzzy implication among others under consideration, by evaluating the metric distance between each implication and the ideal implication for a given data application. This approach has been tested on a case of five FI with promising results.

The ninth paper is entitled "From product recommendation to cyber-attack prediction: Generating attack graphs and predicting future attacks". The authors are Nikolaos Polatidis from the University of Brighton UK, Elias Pimenidis from the University of the West of England Bristol, UK, Michalis Pavlidis from the University of Brighton UK, Spyridon Papastergiou, from the University of Piraeus, Greece and Haralambos Mouratidis from the University of Brighton UK. This interesting research paper proposes an approach that employs attack graphs using data supplied from the maritime supply chain infrastructure. All possible paths capable to be exploited in order to gain access have been delivered. Moreover, a recommendation system has been utilized to predict future attack steps within a network. Overall, the authors propose the use of a recommender system in the prediction of cyber-attacks.

Experiments have shown a potential good practical application.

The tenth paper is authored by Georgios Drakopoulos, from the Ionian University, Corfu, Greece, Foteini Stathopoulou from the University of Luxembourg, Andreas Kanavos from the Hellenic Open University, Patras, Greece, Michael Paraskevas and Giannis Tzimas, from the Technological and Educational Institute of Western Greece, Patras, Greece, Phivos Mylonas from the Ionian University, Corfu, Greece and Lazaros Iliadis from the Democritus University of Thrace, Greece. It is entitled "A genetic algorithm for spatiosocial tensor clustering". This interesting research, introduces an enhanced version of a genetic algorithm which has been tailored for community discovery structure in tensors that comprise of spatiosocial data, mainly of linguistic and geolocation type. Both the objective and the chromosome fitness functions have been developed to consider elements of linguistic propagation models. The genetic operators of selection, crossover, and mutation as well as the newly added double mutation operator, are functioning directly on the community level. Several approaches for maintaining gene variability across generations have been examined in an extensive simulation powered by Google TensorFlow.

The title of the eleventh paper is "An efficient classification approach in imbalanced datasets for intrinsic plagiarism detection". The authors are Andrianna Polydouri, Eleni 
Vathi, Georgios Siolas, and Andreas Georgios Stafylopatis, from the National Technical University of Athens, Greece. This research discusses the introduction of an intrinsic plagiarism detection system. A supervised machine learning classification model has been employed. It is the first time that the problem of imbalanced data is considered as a crucial parameter with important effect on this task. Several experiments have been performed with various balancing techniques in order to overcome this limitation. Moreover, novel stylistic features have been taken into consideration. The authors combine their features and imbalanced dataset treatment with various classification methods. The proposed system has been successfully tested on the data corpora of PAN Webis intrinsic plagiarism detection shared tasks.

The twelfth paper is entitled "Mutual Information Algorithms for Optimal Attribute Selection in Data Driven Partitions of Databases". The authors are Ioannis Stephanakis from the Hellenic Telecommunication Organization, Theodoros Iliou and George Anastassopoulos from the Democritus University of Thrace, Greece. The research presented herein is related to an approach capable of representing complex datasets, as a minimal set of coherent attribute sets of reduced dimensions. The novelty of this paper is in the employment of piecewise analysis of compact clusters in order to increase overall Shannon's mutual informationentropy as a variant to conventional Classification and Regression Trees (CART). Numerical data regarding a testbed system for anomaly detection have been provided in order to illustrate the aforementioned approach.
The final thirteenth paper has the title "Sonar Inspired Optimization (SIO) in Engineering Applications". SIO is a novel Nature Inspired Intelligent scheme which was inspired by the SONAR mechanism, which is used by Warships to detect targets and avoid mines. This research, introduces improvements to this approach in an attempt to increase its performance. Results from experiments in known constrained Engineering applications are presented and discussed. SIO tackles with these problems, managing to overcome the performance of other Nature Inspired metaheuristics, heuristics and mathematical approaches.

We wish to express our appreciation and gratitude to the Editors in Chief of the Springer Evolving Systems Journal Professors P. Angelov, D. Filev, N. K. Kasabov, for their full support and for offering us their high quality journal for this Special Issue.

We hope that this Special Issue will be a significant contribution to the existing literature. We aim in inspiring further research on the development of Evolving Systems' algorithms and applications in all timely scientific domains.

Publisher's Note Springer Nature remains neutral with regard to jurisdictional claims in published maps and institutional affiliations. 